\title{
Viable Solutions for a Class of Delay Evolution Problems
}

\author{
Moufida Amiour, Mustapha Fateh Yarou * \\ LMPA Laboratory, Department of Mathematics, Jijel University, Algeria
}

\begin{abstract}
In this paper, we give existence results for viable solutions in the so-called fully constrained set for functional differential inclusions in Banach spaces for a non-autonomous set-valued mapping with convex compact values. We study also the time dependent case of these invariance sets.
\end{abstract}

Keywords Differential Inclusion, Delay, Viability, Invariance Set, Absolutely continuous solution.

AMS 2010 subject classifications 34K09, 28A05

DOI: $10.19139 /$ soic.v7i2.334

\section{Introduction}

Let $E$ be a separable Banach space, $\mathcal{C}_{0}=\mathcal{C}([-r, 0], E)$ the space of continuous functions from $[-r, 0]$ to $E$. For any $T>0, x:[-r, T] \longrightarrow E$ and $t \in[0, T]$, define the function $x_{t} \in \mathcal{C}_{0}$ by

$$
x_{t}(s)=\tau(t) x(s)=x(t+s) \quad \forall s \in[-r, 0]
$$

and consider the functional differential inclusion

$$
\left\{\begin{array}{lr}
\dot{x}(t) \in F\left(t, x_{t}\right) & \text { a.e. } t \in[0, T] \\
\tau(0) x=x_{0}=\varphi & \text { on }[-r, 0],
\end{array}\right.
$$

where $F$ is a set-valued function from $[0, T] \times \mathcal{C}_{0}$ with nonempty convex compact values in $E$, and $\varphi \in \mathcal{C}_{0}$. The existence of viable solutions for such problems with memory has been studied by several authors ([4], [5], [10], [12], [13], [14]). This class of problems is motivated by the evolution of control systems with feedbacks, dynamic evolutions and planning procedures in microeconomics, for more details and examples, see [1]. In [13], the existence of solutions in the invariance set $E_{0}=\left\{\varphi \in \mathcal{C}_{0}, \varphi(0) \in D\right\}$ where $D$ is closed convex nonempty in $E$, has been established. The purpose of this paper is to show the existence of viable solutions in a set more natural introduced by [11] for a functional differential equations and called the fully constrained set: $E_{D}=\{\varphi \in$ $\left.\mathcal{C}_{0}, \varphi(s) \in D, \forall s \in[-r, 0]\right\}$. The following simple example (see [11]) shows that conditions of invariance for sets $E_{0}$ and $E_{D}$, may be different for the same problem. Consider the problem (1) with

$$
F\left(t, x_{t}\right)=\left\{a x_{t}(0)+b x_{t}(-r)\right\}=\{a x(t)+b \tau(-r) x(t)\}, \quad t \geq 0,
$$

where $a$ and $b$ are real constants. Let $D=\{c\}, c \in \mathbb{R}^{*}$. The conditions for invariance of $E_{0}$ and $E_{D}$ are respectively

$$
a \varphi(0)+b \varphi(-r)=0, \quad \varphi \in E_{0}
$$
*Correspondence to: Mustapha Fateh Yarou (Email: mfyarou@yahoo.com). LMPA Laboratory, Department of Mathematics, Jijel
University, PB98, Cite Ouled Aissa, Algeria (18000).

ISSN 2310-5070 (online) ISSN 2311-004X (print)

Copyright (C) 2019 International Academic Press 


$$
a \varphi(0)+b \varphi(-r)=0, \quad \varphi \in E_{D}
$$

In the equation (2) the value $\varphi(-r)$ could be any we want. Hence the condition (2) is satisfied if and only if $a=b=0$, while the condition (3) holds if and only if $a+b=0$.

To establish the main result, we adopt a discretization approach; we first obtain the invariance result for an intermediary set: $E_{s}=\left\{\varphi \in \mathcal{C}_{0}, \varphi(s) \in D\right\}$ for any $s$ fixed in $[-r, 0]$. Next, we give the result for $E_{D}=\bigcap_{r \leq s \leq 0} E_{s}$; we study also the case of variable constraint $D=\Gamma(t)$, where $\Gamma$ is a set-valued function with closed graph. Our approach is different from that of [11] since our method consists in transforming the delayed problem to a problem without delay and applying the results known in this case. Moreover, we weaken the regularity assumptions existing for this type of problems thanks to a set-valued version of Scorza-Dragoni Theorem.

\subsection{Contributions}

The main contribution of this paper is a generalization of existence results for viable solutions of differential inclusions with delay. We consider a more natural and general invariance set, called the "fully constrained set", as well for the case of a fixed set as of the variable case. Our results generalize those in [13], [11] and [3].

\subsection{Organization}

The rest of this paper is organized as follows. In Section 2, we present the basic notions for set-valued mappings and functional differential inclusions. In Section 3 , we give first a weak version for the existence of viable solutions to first order differential inclusion without delay. This result is used to extend the discretization approach to solve functional differential inclusions for a general and natural invariance set. Finally, the variable case of viability set is studied in Section 4.

\section{Notations and preliminaries}

Throughout the paper $E$ will denote a separable Banach space with norm $\|\|,$.$D a nonempty closed convex set of$ $E$ and $\mathcal{C}_{t}=\mathcal{C}([-r, t], E)$ the set of continuous functions from $[-r, t]$ to $E$, equipped with the topology of uniform convergence. Clearly, if $x \in \mathcal{C}_{T}$, then $x_{t} \in \mathcal{C}_{0}$ and the mapping $x \longmapsto x_{t}$ is continuous from $\mathcal{C}_{T}$ to $\mathcal{C}_{0}$ in the sense of uniform convergence. For $r>0$ and $s \in[-r, 0]$, let define the set $E_{s}=\left\{\varphi \in \mathcal{C}_{0}, \varphi(s) \in D\right\}$, equipped with the topology induced by the norm

$$
\|\varphi\|_{s}=\sup _{-r \leq \tau \leq 0}\|\varphi(\tau)\|, \varphi \in E_{s} .
$$

We denote by $c(E)$ (resp. $c k(E)$ ) the set of nonempty closed (resp. convex compact) subsets of $E, \lambda$ the Lebesgue measure on $\mathbb{R}, \tau_{\lambda}(I)$ the $\sigma$-algebra of measurable subsets of $I \subset \mathbb{R}$, and $\mathcal{B}(D)$ the class of Borel measurable nonempty subsets of $D$.

A function $y: I=[0, T] \longrightarrow E$ is said absolutely continuous if there exists a Lebesgue-Bochner integrable function $\dot{y}$ from $I$ to $E$ such that $y(t)=y(0)+\int_{0}^{t} \dot{y}(s) d s$. A set-valued function $F: I \longrightarrow c k(E)$ is scalarly $\lambda$ measurable if for any $x^{\prime}$ in the dual $E^{\prime}$ of $E$ the scalar function $\delta^{\star}\left(x^{\prime}, F(\cdot)\right)$ is $\lambda$-measurable, $\delta^{\star}(\cdot, K)$ is the support function of $K$, that is, for all $x^{\prime} \in E^{\prime}$,

$$
\delta^{\star}\left(x^{\prime}, K\right)=\sup _{x \in K}\left\langle x^{\prime}, x\right\rangle
$$

For a closed convex subset $K$, the distance function is defined by

$$
d(x, K)=\sup _{x^{\prime} \in E^{\prime}}\left(\left\langle x^{\prime}, x\right\rangle-\delta^{\star}\left(x^{\prime}, K\right)\right) .
$$

A set-valued function $G$ from a topological space $S$ to $c k(E)$ is upper semi-continuous if for any closed subset $U$ of $E,\{x \in S: G(x) \cap U \neq \emptyset\}$ is closed. If $G$ is upper semi-continuous, then $G$ is scalarly upper semi-continuous, that 
is, for any $x^{\prime} \in E^{\prime}$, the scalar function $\delta^{\star}\left(x^{\prime}, G(\cdot)\right)$ is upper semi-continuous. Further, any upper semi-continuous set-valued function has closed graph.

The Bouligand tangent cone of $D$ at the point $x$ is defined by

$$
T_{D}(x)=\left\{y \in E: \liminf _{h \longrightarrow 0^{+}} h^{-1} d(x+h y, D)=0\right\} .
$$

If $D$ is convex, then $T_{D}(x)$ is convex. We refer to [8] and [9] for details concerning measurable set-valued functions. We end this section with the following result due to [7].

\section{Theorem 1}

Let $D$ be a closed convex nonempty subset of $E$ and $F$ be a set-valued function defined on $[0, T] \times D$ with values in $c k(E)$ verifying

(i) $F$ is $\tau_{\lambda}([0, T]) \otimes \mathcal{B}(D)$-measurable,

(ii) for any $t$ fixed in $[0, T], F(t, \cdot)$ is upper semi-continuous on $D$,

(iii) there exists a balanced set $K \in \operatorname{ck}(E)$ such that for all $(t, x) \in[0, T] \times D$,

$$
F(t, x) \subset(1+\|x\|) K
$$

(iv) $F(t, x) \cap T_{D}(x) \neq \emptyset, \quad \forall(t, x) \in[0, T] \times D$.

Then, for any $x_{0} \in D$, there exists an absolutely continuous function $x:[0, T] \longrightarrow E$, verifying

$$
\left\{\begin{array}{l}
\dot{x}(t) \in F(t, x(t)) \text { a.e. on }[0, T] \\
x(t) \in D, \forall t \in[0, T] \\
x(0)=x_{0} .
\end{array}\right.
$$

\section{Fully constrained sets}

We shall say that a continuous function $x:[-r, T] \longrightarrow E$ is a solution of

$$
\dot{x}(t) \in F\left(t, x_{t}\right) \text { a.e. } t \in[0, T]
$$

with the initial value $\varphi \in \mathcal{C}_{0}$ if $\tau(0) x=\varphi$ on $[-r, 0]$ and $x$ is an absolutely continuous function on $[0, T]$, which verifies (6) almost everywhere on $[0, T]$. Let $A$ be a subset of $\mathcal{C}_{0}$, we shall say that a solution $x$ of (6) is viable in $A$ if $x_{t} \in A$ for all $t \in[0, T] . A$ is said to be strongly invariant if for any initial function $\varphi \in A$, all solutions are viable in $A$.

First, let state a weak version of Theorem 1 by using a set-valued version of Scorza-Dragoni Theorem and the Dugundji Theorem.

Proposition 1

Let $D$ be a closed convex nonempty subset of $E, F$ a set-valued function defined on $[0, T] \times D$ with values in $c k(E)$ verifying

(i) $\forall x \in D, t \mapsto F(t, x)$ is measurable,

(ii) $\forall t \in[0, T], x \mapsto F(t, x)$ is upper semi-continuous on $D$,

(iii) there exists a balanced set $K \in \operatorname{ck}(E)$ such that for all $(t, x) \in[0, T] \times D$,

$$
F(t, x) \subset(1+\|x\|) K
$$

(iv) $F(t, x) \cap T_{D}(x) \neq \emptyset, \quad \forall(t, x) \in[0, T] \times D$.

Then, for any $x_{0} \in D$, there exists an absolutely continuous function $x:[0, T] \longrightarrow E$, verifying

$$
\left\{\begin{array}{l}
\dot{x}(t) \in F(t, x(t)) \text { a.e. on }[0, T] \\
x(t) \in D, \forall t \in[0, T] \\
x_{0}=x(0) .
\end{array}\right.
$$


Proof: Using the Scorza-Dragoni's Theorem (see [6]), there is a set-valued function $F_{0}:[0, T] \times D \longrightarrow c k(E)$, which is globally measurable and has the following properties:

(1) There is a set $N \subset[0, T]$, independent of $(t, x)$ such that $\lambda(N)=0$ and

$$
F_{0}(t, x) \subset F(t, x), \text { for all } t \in[0, T] \backslash N \text { and for all } x \in D ;
$$

(2) if $x, z:[0, T] \longrightarrow E$ are measurable mappings with $z(t) \in F(t, x(t))$ a.e., then $z(t) \in F_{0}(t, x(t))$ a.e.

(3) for every $\varepsilon>0$, there is a compact subset $J_{\varepsilon} \subset[0, T]$ such that $\lambda\left([0, T] \backslash J_{\varepsilon}\right)<\varepsilon$, the restriction of $F_{0}$ on $J_{\varepsilon} \times D$ is upper semi-continuous and

$$
\emptyset \neq F_{0}(t, x) \subset F(t, x) ; \text { for all }(t, x) \in J_{\varepsilon} \times D .
$$

By property (3), there exists a sequence of compact sets $J_{\varepsilon} \subset[0, T]$ with $\lambda\left([0, T] \backslash J_{\varepsilon}\right)=\varepsilon_{n} \underset{n \rightarrow \infty}{\longrightarrow} 0$ such that the restriction of $F_{0}$ to $J_{\varepsilon} \times D$ is upper semi-continuous and has nonempty values. We may also assume that $\left(J_{\varepsilon}\right)$ is increasing. By Dugundji's Theorem (see [2]), there is an upper semi-continuous extension $\tilde{F}_{n}$ of $F_{0} / J_{\varepsilon} \times D$, and

$$
\tilde{F}_{n}(t, x) \subset(1+\|x\|) K .
$$

Since $T_{D}(x)$ is convex and $F_{0}$ satisfies the condition $(i v)$, it is therefore obvious that $\tilde{F}_{n}$ satisfies $(i v)$ too.

So $\tilde{F}_{n}$ satisfies the hypotheses of Theorem 1 . Thus, for every $x_{0} \in D$ there exists an absolutely continuous function $x_{n}:[0, T] \longrightarrow E$ such that

$$
\left\{\begin{array}{l}
\dot{x}_{n}(t) \in \tilde{F}_{n}\left(t, x_{n}(t)\right) \text { a.e. on }[0, T] \\
x_{n}(t) \in D \forall t \in[0, T] \\
x_{n}(0)=x_{0}
\end{array}\right.
$$

By (8) and (9) we have for almost every $t$ in $[0, T]$,

$$
\dot{x}_{n}(t) \in \tilde{F}_{n}\left(t, x_{n}(t)\right) \subset\left(1+\left\|x_{n}(t)\right\|\right) K .
$$

Since $K$ is compact, there is a strictly positive number $M$ such that

$$
\tilde{F}_{n}\left(t, x_{n}(t)\right) \subset\left(1+\left\|x_{n}(t)\right\|\right) M \bar{B}_{E},
$$

where $\bar{B}_{E}$ is the closed united ball. Moreover, $x_{n}$ is absolutely continuous on $[0, T]$, then

$$
\left\|x_{n}(t)-x_{n}(0)\right\| \leq \int_{0}^{t} M\left(1+\left\|x_{n}(w)\right\|\right) d w \quad \forall t \in[0, T] .
$$

By applying the Gronwall inequality, we conclude that for all $\mathrm{t}$

$$
\left\|x_{n}(t)\right\| \leq\left(\left\|x_{n}(0)\right\|+M T\right) e^{M T}
$$

and then, for almost every $\mathrm{t}$ in $[0, \mathrm{~T}]$

$$
\dot{x}_{n}(t) \in \tilde{F}_{n}\left(t, x_{n}(t)\right) \subset(1+l) K
$$

with $l=\left(\left\|\varphi_{0}(0)\right\|+M T\right) e^{M T}$. Since $K$ is convex compact and from (10), $\left(\dot{x}_{n}\right)_{n}$ is relatively compact in $L_{E}^{1}([0, T])$. Thus we can extract a subsequence still denoted $\left(\dot{x}_{n}\right)_{n}$ for simplicity which converges $\sigma\left(L^{1}, L^{\infty}\right)$ to a function $y \in L_{E}^{1}$. Furthermore, since $\left(x_{n}\right)$ is absolutely continuous on $[0, T]$ and by using (10), we obtain

$$
\left\|\dot{x}_{n}(t)\right\| \leq(1+l) M,
$$

the sequence is equi-continuous, and

$$
\forall t \in[0, T],\left(x_{n}(t)\right)_{n} \subset x_{n}(0)+T(1+l) K .
$$


By Ascoli's Theorem, $\left(x_{n}\right)_{n}$ converges uniformly on $[0, T]$ to $x$ with

$$
x(t)=x(0)+\int_{0}^{t} y(w) d w \quad \forall t \in[0, T]
$$

so $\dot{x}(t)=y(t)$ a.e., since $D$ is closed,

$$
x(t) \in D \quad \forall t \in[0, T] .
$$

We will show that for all $t \in[0, T], \quad \dot{x}(t) \in F(t, x(t))$ almost everywhere.

As $\left(x_{n}\right),\left(\dot{x}_{n}\right)$ are two measurable mappings satisfying

$$
\dot{x}_{n}(t) \in \tilde{F}_{n}(t, x(t)) \text { a.e. }
$$

then by property (2), for all $n \in \mathbb{N}$, there is a Lebesgue null set $N_{n} \subset J_{n}$ such that

$$
\dot{x}_{n}(t) \in F_{0}\left(t, x_{n}(t)\right) \quad \forall t \in J_{n} \backslash N_{n} .
$$

Let $N_{0}=\left([0, T] \backslash \cup_{n} J_{n}\right) \cup\left(\cup_{n} N_{n}\right)$ which is Lebesgue-negligible. Indeed,

$$
\begin{gathered}
\lambda\left(N_{0}\right)=\lambda\left(\left([0, T] \backslash \cup_{n} J_{n}\right) \cup\left(\cup_{n} N_{n}\right)\right) \\
\leq \lambda\left(\left([0, T] \backslash \cup_{n} J_{n}\right)+\lambda\left(\cup_{n} N_{n}\right)\right. \\
\leq\left(\cap_{n}\left([0, T] \backslash J_{n}\right)\right)+\sum_{n} \lambda\left(N_{n}\right),
\end{gathered}
$$

we have that the set $J_{n}$ has finite measure and the sequence $\left([0, T] \backslash J_{n}\right)$ is decreasing because $\left(J_{n}\right)$ is increasing, thus

$$
\lambda\left(\cap_{n}\left([0, T] \backslash J_{n}\right)\right)=\lim _{n \longrightarrow \infty}\left(\lambda\left([0, T] \backslash J_{n}\right)\right)=\lim _{n \longrightarrow \infty} \varepsilon_{n}=0
$$

and therefore

$$
\lambda\left(N_{0}\right) \leq \lim _{n \longrightarrow \infty} \lambda\left([0, T] \backslash J_{n}\right)+\sum_{n} \lambda\left(N_{n}\right)=0 .
$$

For all $t \in[0, T] \backslash N_{0}$, there is an integer $n_{0}=n_{0}(t) \in N$ such that for all $n \geq n_{0}, t \in J_{n} \backslash N_{n}$, so by the relation (11), we obtain

$$
\dot{x}(t) \in F_{0}(t, x(t)), \quad \forall n \geq n_{0} .
$$

On the other hand, since $F_{0}$ is upper semi-continuous on $J_{n} \times D$ and $x_{n}(t) \longrightarrow x(t)$ when $n \longrightarrow \infty$, it follows that for all $x^{\prime} \in E^{\prime}$

$$
\limsup _{n \longrightarrow \infty} \delta^{\star}\left(x^{\prime}, F_{0}\left(t, x_{n}(t)\right)\right) \leq \delta^{\star}\left(x^{\prime}, F_{0}(t, x(t))\right) .
$$

For $t \notin N_{0}$ and $n \geq n_{0}$, we have

$$
\left\langle x^{\prime}, \dot{x}_{n}(t)\right\rangle \leq \delta^{\star}\left(x^{\prime}, F_{0}(t, x(t))\right)
$$

thus

$$
\limsup _{n \longrightarrow \infty}\left\langle x^{\prime}, \dot{x}_{n}(t)\right\rangle \leq \limsup _{n \longrightarrow \infty} \delta^{\star}\left(x^{\prime}, F_{0}\left(t, x_{n}(t)\right)\right) \leq \delta^{\star}\left(x^{\prime}, F_{0}(t, x(t))\right),
$$

by Fatou's lemma we deduce that for every measurable set $B \subset[0, T]$ and every $x^{\prime} \in E^{\prime}$,

$$
\begin{gathered}
\int_{B}\left\langle x^{\prime}, \dot{x}(t)\right\rangle d t=\lim _{n \longrightarrow \infty} \int_{B}\left\langle x^{\prime}, \dot{x}_{n}(t)\right\rangle d t \\
=\limsup _{n \longrightarrow \infty} \int_{B}\left\langle x^{\prime}, \dot{x}_{n}(t)\right\rangle d t \\
\leq \int_{B} \limsup _{n \longrightarrow \infty}\left\langle x^{\prime}, \dot{x}_{n}(t)\right\rangle d t
\end{gathered}
$$




$$
\leq \int_{B} \delta^{\star}\left(x^{\prime}, F_{0}(t, x(t))\right) d t
$$

So

$$
\left\langle x^{\prime}, \dot{x}(t)\right\rangle \leq \delta^{\star}\left(x^{\prime}, F_{0}(t, x(t))\right) \text { a.e. }
$$

then

$$
\sup _{x^{\prime} \in E^{\prime}}\left\langle x^{\prime}, \dot{x}(t)\right\rangle-\delta^{\star}\left(x^{\prime}, F_{0}(t, x(t))\right) \leq 0
$$

since $F_{0}$ has closed convex values, by (4) we get $d\left(\dot{x}(t), F_{0}(t, x(t))\right)=0$, which implies that $\dot{x}(t) \in F_{0}(t, x(t))$ a.e. By property (1),

$$
\dot{x}(t) \in F(t, x(t)), \quad \forall t \in[0, T] \backslash N_{0},
$$

which shows that

$$
\dot{x}(t) \in F(t, x(t)) \quad \text { a.e. } t \in[0, T] .
$$

Now, let's give the existence of viable solutions for $E_{s}, s \in[-r, 0]$.

\section{Theorem 2}

For $s$ fixed in $[-r, 0]$, let $D$ be a closed convex nonempty subset of $E$ and $F$ a set-valued function defined on $[0, T] \times \mathcal{C}_{0}$ with values in $c k(E)$ verifying

(i) $\forall \theta \in E_{s}, t \mapsto F(t, \theta)$ is measurable,

(ii) $\forall t \in[0, T], \theta \mapsto F(t, \theta)$ is upper semi-continuous on $E_{s}$,

(iii) there exists a balanced set $K \in \operatorname{ck}(E)$ such that for all $(t, \theta) \in[0, T] \times E_{0}$,

$$
F(t, \theta) \subset(1+\|\theta(0)\|) K
$$

(iv) $F(t, \theta) \cap T_{D}(\theta(0)) \neq \emptyset, \quad \forall(t, \theta) \in[0, T] \times E_{0}$.

Then, for any $\varphi_{0} \in E_{s}$, there exists a continuous function $x^{s}:[-r, T] \longrightarrow E$, absolutely continuous on $[0, T]$, verifying

$$
\left\{\begin{array}{l}
\dot{x}^{s}(t) \in F\left(t, \tau(t-s) x^{s}\right) \quad \text { a.e } \quad \text { on }[0, T] \\
\tau(t-s) x^{s}=x_{t-s}^{s} \in E_{s}, \quad \forall t \in[0, T] \\
x^{s}(t)=\varphi_{0}(t) \quad \forall t \in[-r, 0] .
\end{array}\right.
$$

Proof: Let $\mathcal{P}_{n}$ be a subdivision of $[0, T]$ defined by

$$
\mathcal{P}_{n}=\left\{t_{i}^{n}=T \frac{i}{2^{n}}, \quad i=0,1, \ldots, 2^{n}\right\}
$$

Let $x \in D$, we define $f_{1}^{x}:\left[-r, t_{1}^{n}\right] \longrightarrow E$ by

$$
f_{1}^{x}(u)=\left\{\begin{array}{lc}
\varphi_{0}(u) & u \in[-r, s] \\
\varphi_{0}(s)+\frac{u-s}{2^{-n} T}\left(x-\varphi_{0}(s)\right) & u \in\left[s, s+t_{1}^{n}\right] \\
x & u \in\left[s+t_{1}^{n}, t_{1}^{n}\right]
\end{array}\right.
$$

and $\tau\left(t_{1}^{n}\right): \mathcal{C}_{t_{1}^{n}} \longrightarrow \mathcal{C}_{0}, f_{1}^{x} \longmapsto \tau\left(t_{1}^{n}\right) f_{1}^{x}:$

$$
\tau\left(t_{1}^{n}\right) f_{1}^{x}(u)=f_{1}^{x}\left(u+t_{1}^{n}\right) \quad u \in[-r, 0] .
$$

Obviously, we have $f_{1}^{x} \in \mathcal{C}_{t_{1}^{n}}, \tau\left(t_{1}^{n}\right) f_{1}^{x}(0)=x \in D$, so $\tau\left(t_{1}^{n}\right) f_{1}^{x} \in E_{s} \subset \mathcal{C}_{0}$.

Moreover, the function $x \longmapsto \tau\left(t_{1}^{n}\right) f_{1}^{x}$ is 1-Lipschitz:

$$
\left\|\tau\left(t_{1}^{n}\right) f_{1}^{x}-\tau\left(t_{1}^{n}\right) f_{1}^{y}\right\|_{s}=\sup _{-r \leq u \leq s}\left\|f_{1}^{x}\left(u+t_{1}^{n}\right)-f_{1}^{y}\left(u+t_{1}^{n}\right)\right\|
$$




$$
\begin{gathered}
=\sup _{s-t_{1}^{n} \leq u \leq s}\left\|\frac{u+t_{1}^{n}-s}{2^{-n} T}(x-y)\right\| \\
=\|x-y\| .
\end{gathered}
$$

Now, define the set-valued function $S_{1}^{n}:\left[0, t_{1}^{n}\right] \times D \longrightarrow c k(E)$ by:

$$
S_{1}^{n}(t, x)=F\left(t, \tau\left(t_{1}^{n}\right) f_{1}^{x}\right) \quad \forall(t, x) \in\left[0, t_{1}^{n}\right] \times D .
$$

Since $F$ is measurable and $x \longmapsto \tau\left(t_{1}^{n}\right) f_{1}^{x} 1$-Lipschitz, $S_{1}^{n}(\cdot, x)$ is obviously measurable. Also, $S_{1}^{n}(t, \cdot)$ is upper semi-continuous on $D$ because $F(t, \cdot)$ is so on $E_{s}, \forall t \in[0, T]$. Moreover, conditions (iii) and (iv) give for any $t \in\left[0, t_{1}^{n}\right]$ and $x$ in $D$,

$$
S_{1}^{n}(t, x)=F\left(t, \tau\left(t_{1}^{n}\right) f_{1}^{x}\right) \subset\left(1+\left\|f_{1}^{x}\left(t_{1}^{n}\right)\right\|\right) K=(1+\|x\|) K,
$$

and

$$
S_{1}^{n}(t, x) \cap T_{D}(x)=F\left(t, \tau\left(t_{1}^{n}\right) f_{1}^{x}\right) \cap T_{D}\left(\tau\left(t_{1}^{n}\right) f_{1}^{x}(0)\right) \neq \emptyset .
$$

So $S_{1}^{n}$ verifies hypotheses of Proposition 1 , then for any $\varphi_{0} \in D$ there exists an absolutely continuous function $x_{1}^{n}$ from $\left[0, t_{1}^{n}\right]$ to $E$ such that

$$
\left\{\begin{array}{l}
\dot{x}_{1}^{n}(t) \in S\left(t, x_{1}^{n}(t)\right) \text { a.e. on }\left[0, t_{1}^{n}\right] \\
x_{1}^{n}(t)=\varphi_{0}(0)+\int_{0}^{t} \dot{x}_{1}^{n}(w) d w \forall t \in\left[0, t_{1}^{n}\right] \\
x_{1}^{n}(t) \in D, \forall t \in\left[0, t_{1}^{n}\right] \\
x_{1}^{n}(0)=\varphi_{0}(0)
\end{array}\right.
$$

So $x_{1}^{n}$ verifies

$$
\dot{x}_{1}^{n}(t) \in F\left(t, \tau\left(t_{1}^{n}\right) f_{1}^{x_{1}^{n}(t)}\right) \quad \text { a.e } \quad t \in\left[0, t_{1}^{n}\right] .
$$

Put

$$
x_{n}^{s}(t)= \begin{cases}\varphi_{0}(t) & \forall t \in[-r, 0] \\ x_{1}^{n}(t) & \forall t \in\left[0, t_{1}^{n}\right] .\end{cases}
$$

Then, $x_{n}^{s}:\left[-r, t_{1}^{n}\right] \longrightarrow E, x_{n}^{s}$ absolutely continuous on $\left[0, t_{1}^{n}\right], x_{n}^{s}(t) \in D$.

Similarly, for any $x \in D$, we define $f_{2}^{x}:\left[-r, t_{2}^{n}\right] \longrightarrow E$

$$
f_{2}^{x}(u)=\left\{\begin{array}{lc}
x_{n}^{s}(u-s) & u \in\left[-r, t_{1}^{n}+s\right] \\
x_{n}^{s}\left(t_{1}^{n}\right)+\frac{u-t_{1}^{n}-s}{2^{-n} T}\left(x-x_{n}^{s}(t)\right) & u \in\left[t_{1}^{n}+s, t_{2}^{n}+s\right] \\
x & u \in\left[s+t_{2}^{n}, t_{2}^{n}\right]
\end{array}\right.
$$

and $\tau\left(t_{2}^{n}\right): \mathcal{C}_{t_{2}^{n}} \longrightarrow \mathcal{C}_{0}, \tau\left(t_{2}^{n}\right) f_{2}^{x}(0)=f_{2}^{x}\left(t_{2}^{n}\right)=x \in D$, so $\tau\left(t_{2}^{n}\right) f_{2}^{x} \in E_{s}$ and $x \longmapsto \tau\left(t_{2}^{n}\right) f_{2}^{x}$ is 1-lipschitz:

$$
\left\|\tau\left(t_{2}^{n}\right) f_{2}^{x}-\tau\left(t_{2}^{n}\right) f_{2}^{y}\right\|_{s}=\sup _{s-2^{n} \leq u \leq s}\left\|\frac{u+t_{2}^{n}-t_{1}^{n}-s}{2^{n} T}(x-y)\right\|=\|x-y\| .
$$

Define again $S_{2}^{n}:\left[t_{1}^{n}, t_{2}^{n}\right] \times D \longrightarrow c k(E)$

$$
S_{2}^{n}(t, x)=F\left(t, \tau\left(t_{2}^{n}\right) f_{2}^{x}\right) \quad \forall(t, x) \in\left[t_{1}^{n}, t_{2}^{n}\right] \times D .
$$

Also, the hypotheses on $F$ ensure that $S_{2}^{n}(., x)$ is measurable, $S_{2}^{n}(t, \cdot)$ upper semi-continuous on $D, \forall t \in[0, T]$.

$$
S_{2}^{n}(t, x) \subset(1+\|x\|) K, \quad \forall(t, x) \in\left[t_{1}^{n}, t_{2}^{n}\right] \times D
$$

and

$$
S_{2}^{n}(t, x) \cap T_{D}(x) \neq \emptyset .
$$


Then, there exists an absolutely continuous function $x_{2}^{n}$ from $\left[t_{1}^{n}, t_{2}^{n}\right]$ on $E$ such that

$$
\left\{\begin{array}{l}
\dot{x}_{2}^{n}(t) \in S\left(t, x_{2}^{n}(t)\right) \text { a.e. on }\left[t_{1}^{n}, t_{2}^{n}\right] \\
x_{2}^{n}(t)=x_{n}^{s}\left(t_{1}^{n}\right)+\int_{t_{1}^{n}}^{t} \dot{x}_{2}^{n}(w) d w \forall t \in\left[t_{1}^{n}, t_{2}^{n}\right] \\
x_{2}^{n}(t) \in D \forall t \in\left[t_{1}^{n}, t_{2}^{n}\right] \\
x_{2}^{n}\left(t_{1}^{n}\right)=x_{n}^{s}\left(t_{1}^{n}\right)=x_{1}^{n}\left(t_{1}^{n}\right) .
\end{array}\right.
$$

So $x_{2}^{n}$ verifies

$$
\dot{x}_{2}^{n}(t) \in F\left(t, \tau\left(t_{2}^{n}\right) f_{2}^{x_{2}^{n}(t)}\right) \text { a.e. on }\left[t_{1}^{n}, t_{2}^{n}\right]
$$

Put

$$
x_{n}^{s}(t)=x_{2}^{n}(t) \quad \forall t \in\left[t_{1}^{n}, t_{2}^{n}\right] .
$$

Then, $x_{n}^{s}:\left[-r, t_{2}^{n}\right] \longrightarrow E, x_{n}^{s}$ absolutely continuous on $\left[0, t_{2}^{n}\right], x_{n}^{s}(t) \in D$. By induction, we define a function $x_{n}^{s}:\left[-r, t_{k}^{n}\right] \longrightarrow E, x_{n}^{s}$ absolutely continuous on $\left[0, t_{k}^{n}\right], x_{n}^{s}(t) \in D$ such that

$$
x_{n}^{s}(t)= \begin{cases}\varphi_{0}(t) & \forall t \in[-r, 0] \\ x_{1}^{n}(t) & \forall t \in\left[0, t_{1}^{n}\right] \\ \cdots & \\ x_{k}^{n}(t) & \forall t \in\left[t_{k-1}^{n}, t_{k}^{n}\right]\end{cases}
$$

and

$$
\left\{\begin{array}{l}
\dot{x}_{n}^{s}(t) \in F\left(t, \tau\left(t_{k}^{n}\right) f_{k}^{x_{n}^{s}(t)}\right) \text { a.e. on }\left[t_{k-1}^{n}, t_{k}^{n}\right] \\
x_{n}^{s}(t)=x_{n}^{s}\left(t_{k-1}^{n}\right)+\int_{t_{k-1}^{n}}^{t} \dot{x}_{n}^{s}(w) d w \forall t \in\left[t_{k-1}^{n}, t_{k}^{n}\right] \\
x_{n}^{s}(t) \in D \forall t \in\left[t_{k-1}^{n}, t_{k}^{n}\right]
\end{array}\right.
$$

and construct a solution on $\left[t_{k}^{n}, t_{k+1}^{n}\right]$.

For any $x \in D$, define $f_{k+1}^{x}:\left[-r, t_{k+1}^{n}\right] \longrightarrow E$ :

$$
\begin{gathered}
f_{k+1}^{x}(u)=\left\{\begin{array}{lc}
x_{n}^{s}(u-s) & u \in\left[-r, t_{k}^{n}+s\right] \\
x_{n}^{s}\left(t_{k}^{n}\right)+\frac{u-t_{k}^{n}-s}{2^{-n} T}\left(x-x_{n}^{s}\left(t_{k}^{n}\right)\right) & u \in\left[t_{k}^{n}+s, t_{k+1}^{n}+s\right] \\
x & u \in\left[s+t_{k+1}^{n}, t_{k+1}^{n}\right]
\end{array}\right. \\
\tau\left(t_{k+1}^{n}\right): \mathcal{C}_{t_{k+1}^{n}} \longrightarrow \mathcal{C}_{0}, \tau\left(t_{k+1}^{n}\right) f_{k+1}^{x}(0)=f_{k+1}^{x}\left(t_{k+1}^{n}\right)
\end{gathered}
$$

$f_{k+1}^{x} \in \mathcal{C}_{t_{k+1}^{n}}, f_{k+1}^{x}\left(t_{k+1}^{n}\right)=x$, so $\tau\left(t_{k+1}^{n}\right) f_{k+1}^{x} \in E_{s}$, and $x \longmapsto \tau\left(t_{k+1}^{n}\right) f_{k+1}^{x}$ is 1-Lipschitz.

Let: $S_{k+1}^{n}:\left[t_{k}^{n}, t_{k+1}^{n}\right] \times D \longrightarrow c k(E)$

$$
S_{k+1}^{n}(t, x)=F\left(t, \tau\left(t_{k+1}^{n}\right) f_{k+1}^{x}\right) .
$$

Then, $S_{k+1}^{n}(\cdot, x)$ is measurable, $S_{k+1}^{n}(t, \cdot)$ is upper semi-continuous on $D$ for any $t \in[0, T]$ and verifies

$$
S_{k+1}^{n}(t, x) \subset(1+\|x\|) K
$$

and

$$
S_{k+1}^{n}(t, x) \cap T_{D}(x) \neq \emptyset .
$$

Then, there exists an absolutely continuous function $x_{k+1}^{n}:\left[t_{k}, t_{k+1}\right] \longrightarrow E$ such that

$$
\left\{\begin{array}{l}
\dot{x}_{k+1}^{n}(t) \in S\left(t, x_{k+1}^{n}(t)\right) \text { a.e. on }\left[t_{k}^{n}, t_{k+1}^{n}\right] \\
x_{k+1}^{n}(t)=x_{n}^{s}\left(t_{k}^{n}\right)+\int_{t_{k}^{n}}^{t} \dot{x}_{k+1}^{n}(w) d w \forall t \in\left[t_{k}^{n}, t_{k+1}^{n}\right] \\
x_{k}^{n}(t) \in D \forall t \in\left[t_{k}^{n}, t_{k+1}^{n}\right] \\
x_{k+1}^{n}\left(t_{k}^{n}\right)=x_{n}^{s}\left(t_{k}^{n}\right) .
\end{array}\right.
$$


Put $x_{n}^{s}(t)=x_{k+1}^{n}(t)$ on $\left[t_{k}^{n}, t_{k+1}^{n}\right]$, and for $t$ in $\left[t_{k}^{n}, t_{k+1}^{n}\left[, \theta_{n}(t)=t_{i}^{n}, \delta_{n}(t)=t_{i+1}^{n}, \theta_{n}(T)=T\right.\right.$. Then $x_{n}^{s}$ is continuous on $[-r, T]$, absolutely continuous on $[0, T]$ such that

$$
\left\{\begin{array}{l}
\dot{x}_{n}^{s}(t) \in F\left(t, \tau\left(\delta_{n}(t)\right) f_{k}^{x_{n}^{s}(t)}\right) \text { a.e. on }[0, T] \\
x_{n}^{s}(t)=\varphi_{0}(0)+\int_{0}^{t} \dot{x}_{n}^{s}(w) d w \forall t \in[0, T] \\
x_{n}^{s}(t) \in D \forall t \in[0, T] \\
x_{n}^{s}(t)=\varphi_{0}(t) \forall t \in[-r, 0]
\end{array}\right.
$$

where for any $t$ in $[0, T], f_{n}^{x^{s}(t)} \in \mathcal{C}_{\delta_{n}(t)}$

$$
f_{n}^{x_{n}^{s}(t)}(u)= \begin{cases}x_{n}^{s}(u-s) & u \in\left[-r, \theta_{n}(t)+s\right] \\ x_{n}^{s}\left(\theta_{n}(t)\right)+\frac{u-\theta_{n}(t)-s}{2^{-n} T}\left(x_{n}^{s}(t)-x_{n}^{s}\left(\theta_{n}(t)\right)\right) \quad u \in\left[\theta_{n}(t)+s, \delta_{n}(t)+s\right] & u \in\left[s+\delta_{n}(t), \delta_{n}(t)\right] .\end{cases}
$$

Consequently, we have obtained a sequence $\left(x_{n}^{s}\right)_{n}$ of continuous functions on $[-r, T]$, absolutely continuous on $[0, T]$, and $\left(f_{n}^{x_{n}^{s}(t)}\right)_{n}$ in $\mathcal{C}_{T}$ verifying (27).

Let us to show that $\left(x_{n}^{s}\right)_{n}$ converge uniformly to an absolutely continuous function on $[0, T]$. By (iii) and (27) we have for almost every $t$ in $[0, T]$, there exists $K \in c k(E)$ such that

$$
\left.\dot{x}_{n}^{s}(t) \in F\left(t, \tau\left(\delta_{n}(t)\right) f_{n}^{x_{n}^{s}(t)}\right) \subset\left(1+\| \tau\left(\delta_{n}(t)\right) f_{n}^{x_{n}^{s}(t)}(0)\right) \|\right) K .
$$

Since $K$ is compact, there is a strictly positive number $M$ such that

$$
F\left(t, \tau\left(\delta_{n}(t)\right) f_{n}^{x_{n}^{s}(t)}\right) \subset\left(1+\left\|x_{n}^{s}(t)\right\|\right) M \bar{B}_{E}
$$

moreover, $x_{n}^{s}$ is absolutely continuous on $[0, T]$, then

$$
\left\|x_{n}^{s}(t)-\varphi_{0}(0)\right\| \leq \int_{0}^{t} M\left(1+\left\|x_{n}^{s}(w)\right\|\right) d w \quad \forall t \in[0, T] .
$$

By applying the Gronwall inequality, we conclude that for all $\mathrm{t}$

$$
\left\|x_{n}^{s}(t)\right\| \leq\left(\left\|\varphi_{0}(0)\right\|+M T\right) e^{M T}
$$

and then, for almost every $\mathrm{t}$ in $[0, \mathrm{~T}]$

$$
\dot{x}_{n}^{s}(t) \in F\left(t, \tau\left(\delta_{n}(t)\right) f_{n}^{x_{n}^{s}(t)}\right) \subset(1+l) K
$$

with $l=\left(\left\|\varphi_{0}(0)\right\|+M T\right) e^{M T}$. Since $K$ is convex compact and from (29), $\left(\dot{x}_{n}^{s}\right)_{n}$ is relatively compact in $L_{E}^{1}([0, T])$. Thus we can extract a subsequence still denoted $\left(\dot{x}_{n}^{s}\right)_{n}$ for simplicity which converges $\sigma\left(L^{1}, L^{\infty}\right)$ to a function $y^{s} \in L_{E}^{1}$. Furthermore, since $\left(x_{n}^{s}\right)$ is absolutely continuous on $[0, T]$ and by using (29), we obtain

$$
\left\|\dot{x}_{n}^{s}(t)\right\| \leq(1+l) M
$$

the sequence is equicontinuous, and for all $t \in[0, T]$,

$$
\left(x_{n}^{s}(t)\right)_{n} \subset \varphi_{0}(0)+T(1+l) K .
$$

By Ascoli's theorem, $\left(x_{n}^{s}\right)_{n}$ converges uniformly on $[0, T]$ to $x^{s}$ with

$$
x^{s}(t)=\varphi_{0}(0)+\int_{0}^{t} y^{s}(w) d w \quad \forall t \in[0, T]
$$


so $\dot{x}^{s}(t)=y^{s}(t)$ a.e., since $D$ is closed,

$$
x^{s}(t) \in D \quad \forall t \in[0, T]
$$

and

$$
\tau(t-s) x^{s}=x_{t-s}^{s} \in E_{s} .
$$

Because $x_{n}^{s}=\varphi_{0}$ on $[-r, 0]$, we can take $x^{s}=\varphi_{0}$ on $[-r, 0]$.

To complete the proof, we'll show that for almost every $t \in[0, T]$,

$$
\lim _{n \longrightarrow \infty} \tau\left(\delta_{n}(t)\right) f_{n}^{x_{n}^{s}(t)}=\tau(t-s) x^{s}
$$

in $E_{s}$.

$$
\begin{gathered}
\left\|\tau\left(\delta_{n}(t)\right) f_{n}^{x_{n}^{s}(t)}-\tau(t-s) x^{s}\right\|_{s}=\sup _{-r \leq u \leq s-2^{-n}}\left\|x_{n}^{s}\left(u+\delta_{n}(t)-s\right)-x_{n}^{s}(u+t-s)\right\| \\
+\sup _{s-2^{-n} \leq u \leq s}\left\|x_{n}^{s}\left(\theta_{n}(t)\right)+\frac{u-s+\theta_{n}(t)-\delta_{n}(t)}{2^{-n} T}\left(x_{n}^{s}(t)-x_{n}^{s}\left(\theta_{n}(t)\right)\right)-x^{s}(u+t-s)\right\| \\
\leq \sup _{-r \leq u \leq s-2^{-n}}\left\|x_{n}^{s}\left(u+\delta_{n}(t)-s\right)-x^{s}\left(u+\delta_{n}(t)\right)\right\|+\sup _{-r \leq u \leq s-2^{-n}}\left\|x^{s}\left(u+\delta_{n}(t)\right)-x^{s}(u+t-s)\right\| \\
+\sup _{s-2^{-n} \leq u \leq s}\left\|\frac{u-s}{2^{-n} T}\left(x_{n}^{s}\left(\theta_{n}(t)\right)-x_{n}^{s}(t)\right)\right\|+\sup _{s-2^{-n} \leq u \leq s}\left\|x_{n}^{s}(t)-x^{s}(u+t-s)\right\| \\
\leq\left\|x_{n}^{s}\left(\theta_{n}(t)\right)-x_{n}^{s}(t)\right\|+\sup _{s-2^{-n} \leq u \leq s}\left\|x^{s}(t)-x^{s}(u+t-s)\right\|+\left\|x_{n}^{s}(t)-x^{s}(t)\right\| .
\end{gathered}
$$

Since

$$
\lim _{n \longrightarrow \infty} \delta_{n}(t)=\lim _{n \longrightarrow \infty} \theta_{n}(t)=t,
$$

$x^{s}$ and $x_{n}^{s}$ are continuous, we conclude that $\tau\left(\delta_{n}(t)\right) f_{n}^{x_{n}^{s}(t)}$ converges to $\tau(t-s) x^{s}$.

Finally, by using a well known closure's theorem (see [8]), we obtain

$$
\left\{\begin{array}{l}
x^{s}(t) \in F\left(t, \tau(t-s) x^{s}\right) \text { a.e. on }[0, T] \\
\tau(t-s) x^{s}=x_{t-s}^{s} \in E_{s} \forall t \in[0, T] \\
x^{s}(t)=\varphi_{0}(t) \forall t \in[-r, 0] .
\end{array}\right.
$$

Remark 1

In assumptions (iii) and $(i v)$ of Theorem 2, we can replace $E_{0}$ by $E_{s}$ for $s$ fixed in $[-r, 0]$, since by construction, we have

$$
\tau\left(\delta_{n}(t)\right) f_{n}^{x_{n}^{s}(t)} \in E_{u} \quad \forall u \in[s, 0]
$$

Then,

$$
\tau\left(\delta_{n}(t) f_{n}^{x_{n}^{s}(t)}\right) \in \bigcap_{s \leq u \leq 0} E_{u}
$$

in particular, $E_{D}=\bigcap_{-r \leq u \leq 0} E_{u}$ then we deduce the following result.

\section{Theorem 3}

Let $E_{D}=\left\{\varphi \in \mathcal{C}_{0}, \varphi(s) \in D, \forall s \in[-r, 0]\right\}$. Suppose that

(i) for all $\theta \in E_{D}, F(t, \theta)$ is measurable,

(ii) for all $t \in[0, T], F(t, \theta)$ upper semi-continuous on $E_{D}$,

(iii) there is a balanced convex compact $K$ in $E$ such that

$$
F(t, \theta) \subset(1+\|\theta(0)\|) K, \quad \forall(t, \theta) \in[0, T] \times E_{0}
$$


(iv) $\quad F(t, \theta) \cap T_{D} \theta(0) \neq \emptyset, \quad \forall(t, \theta) \in[0, T] \times E_{0}$.

Then, $\forall \varphi_{0} \in E_{D}$, there exists a continuous function $x:[-r, T] \longrightarrow E$, absolutely continuous on $[0, T]$, such that

$$
\left\{\begin{array}{l}
\dot{x}(t) \in F(t, \tau(t) x) \text { a.e. on }[0, T] \\
\tau(t-s) x=x_{t-s} \forall t \in[0, T] \\
x(t)=\varphi_{0}(t) \forall t \in[-r, 0]
\end{array}\right.
$$

\section{Case of variable constraint}

In the case of a variable constraint $(D=\Gamma(t))$, we obtain a version of existence theorem by reducing the problem to a problem without delay and applying the following result due to [3].

\section{Theorem 4}

Let $\Gamma$ be a set-valued function with closed graph $G, F: G \longrightarrow c k(E)$ be an upper semi-continuous set-valued function such that $\exists K \in c k(E)$ :

$$
F(t, x) \subset(1+\|x\|) K, \quad \forall(t, x) \in G .
$$

Suppose that for all $t \in[0, T], x \in \Gamma(t)$, and $\varepsilon>0, \exists\left(t_{\varepsilon}, x_{\varepsilon}\right) \in G$ such that

$$
0<t_{\varepsilon}-t \leq \varepsilon, \quad \frac{x_{\varepsilon}-x}{t_{\varepsilon}-t} \in F(t, x)+\varepsilon \overline{B_{E}} .
$$

Then, for all $a \in \Gamma(0)$, there exists an absolutely continuous function $X:[0, T] \longrightarrow E$ such that

$$
\left\{\begin{array}{l}
X(t)=a+\int_{0}^{t} \dot{X}(s) d s \quad \forall t \in[0, T] \\
X(t) \in \Gamma(t) \quad \forall t \in[0, T] \\
\dot{X}(t) \in F(t, X(t)) \text { a.e. on }[0, T] .
\end{array}\right.
$$

Let's define, for any $t \in[0, T]$ and $s \in[-r, 0]$, the variable fully constrained set

$$
H_{s}(t)=\left\{\varphi \in C_{0}: \varphi(s) \in \Gamma(t)\right\} .
$$

We are able to give the existence of viable solution in these sets.

\section{Theorem 5}

Let $\Gamma:[0, T] \longrightarrow c(E)$ be a set-valued function with closed graph, $F:[0, T] \times \mathcal{C}_{0} \longrightarrow c k(E)$ be a globally upper semi-continuous set-valued function (i.e. upper semi-continuous on $[0, T] \times H_{s}(t)$ ) such that there is a balanced convex compact $K \in E$ :

$$
F(t, \varphi) \subset(1+\|\varphi(0)\|) K, \quad \forall(t, \varphi) \in[0, T] \times H_{0}(t) .
$$

Suppose that for all $t \in[0, T], \varepsilon \in H_{0}(t)$, and $\varepsilon>0, \exists\left(t_{\varepsilon}, \varphi_{\varepsilon}\right) \in \operatorname{gr}\left(H_{0}\right)$ such that

$$
0<t_{\varepsilon}-t \leq \varepsilon, \quad \frac{\varphi_{\varepsilon}(0)-\varphi(0)}{t_{\varepsilon}-t} \in F(t, \varphi)+\varepsilon \overline{B_{E}} .
$$

Then, for all $\varphi_{0} \in H_{s}(t)$, there exists an absolutely continuous function $x^{s}:[-r, T] \longrightarrow E$ such that

$$
\left\{\begin{array}{l}
\dot{x}^{s}(t) \in F\left(t, \tau(t-s) x^{s}\right) \text { a.e. on }[0, T] \\
\tau(t-s) x^{s}=x_{t-s}^{s} \in H_{s}(t) \forall t \in[0, T] \\
x^{s}(t)=\varphi_{0}(t) t \in[-r, 0] .
\end{array}\right.
$$


Proof: The proof is the same as that of Theorem 2, the difference being in the condition (iv). We define the subdivision $\mathcal{P}_{n}$, the function $f_{1}^{x}$ and the set-valued function $S_{1}^{n}$ as in (13) and (14). The set-valued function $S_{1}^{n}$ satisfies the tangential condition $\forall t \in[0, T], \forall \varepsilon \in H_{0}(t), \forall \varepsilon>0, \exists\left(t_{\varepsilon}, \varphi_{\varepsilon}\right) \in \operatorname{gr}\left(H_{0}\right)$ such that

$$
0<t_{\varepsilon}-t \leq \varepsilon, \quad \frac{\varphi_{\varepsilon}(0)-\varphi(0)}{t_{\varepsilon}-t} \in F(t, \varphi)+\varepsilon \overline{B_{E}} .
$$

Indeed let $(t, x) \in \operatorname{gr}(\Gamma)$ and assume $\varphi=\tau\left(t_{1}^{n}\right) f_{1}^{x}$. We obtain $\varphi \in \mathcal{C}([-r, 0], E)$ and $\varphi(0)=f_{1}^{x}\left(t_{1}^{n}\right)=x \in \Gamma(t)$ i.e. $\varphi \in H_{0}(t)$.

Let $\varepsilon>0$, by the condition $(i i i)$, there exists $\left(t_{\varepsilon}, \varphi_{\varepsilon}\right) \in \operatorname{gr}\left(H_{0}\right)$ such that

$$
0<t_{\varepsilon}-t \leq \varepsilon, \quad \frac{\varphi_{\varepsilon}(0)-\varphi(0)}{t_{\varepsilon}-t} \in S_{1}^{n}(t, x)+\varepsilon \overline{B_{E}} .
$$

So $S_{1}^{n}$ verifies hypotheses of Theorem 4 , then there exists an absolutely continous function $x_{1}^{n}$ from $\left[0, t_{1}^{n}\right]$ to $E$ such that

$$
\left\{\begin{array}{l}
x_{1}^{n}(t)=\varphi_{0}(0)+\int_{0}^{t} \dot{x}_{1}^{n}(w) d w \quad \forall t \in[0, T] \\
x_{1}^{n}(t) \in \Gamma(t) \quad \forall t \in\left[0, t_{1}^{n}\right] \\
\dot{x}_{1}^{n}(t) \in S_{1}^{n}\left(t, x_{1}^{n}(t)\right) \text { a.e. on }\left[0, t_{1}^{n}\right]
\end{array}\right.
$$

with

$$
\dot{x}_{1}^{n}(t) \in F\left(t, \tau\left(t_{1}^{n}\right) f_{1}^{x_{1}^{n}(t)}\right) \quad \text { a.e. on }\left[0, t_{1}^{n}\right] .
$$

By following the same procedure of the proof of Theorem 2 we deduce that there exists an absolutely continuous function $x_{n}^{s}:[-r, T] \longrightarrow E$ satisfying

$$
\left\{\begin{array}{l}
\dot{x}_{n}^{s}(t) \in F\left(t, \tau\left(t_{k}^{n}\right) f_{k}^{x_{n}^{s}(t)}\right) \text { a.e. on }\left[t_{k-1}^{n}, t_{k}^{n}\right] \\
x_{n}^{s}(t)=x_{n}^{s}\left(t_{k-1}^{n}\right)+\int_{t_{k-1}^{n}}^{t} \dot{x}_{n}^{s}(w) d w \forall t \in\left[t_{k-1}^{n}, t_{k}^{n}\right] \\
x_{n}^{s}(t) \in D \forall t \in\left[t_{k-1}^{n}, t_{k}^{n}\right]
\end{array}\right.
$$

for all $0 \leq k \leq 2^{n}$. For any $t \in[0, T], f_{n}^{x_{n}^{s}(t)} \in \mathcal{C}\left(\left[-r, \delta_{n}(t)\right], E\right)$

$$
f_{n}^{x_{n}^{s}(t)}(u)= \begin{cases}x_{n}^{s}(u-s) & u \in\left[-r, \theta_{n}(t)+s\right] \\ x_{n}^{s}\left(\theta_{n}(t)\right)+\frac{u-\theta_{n}(t)-s}{2^{-n} T}\left(x_{n}^{s}(t)-x_{n}^{s}\left(\theta_{n}(t)\right)\right) & u \in\left[\theta_{n}(t)+s, \delta_{n}(t)+s\right] \\ x_{n}^{s}(t) & u \in\left[\delta_{n}(t)+s, \delta_{n}(t)\right] .\end{cases}
$$

It is clear by construction that $x_{n}^{s}$ is an absolutely continuous functions on $[0, T]$ verifying

$$
\left\{\begin{array}{l}
\dot{x}_{n}^{s}(t) \in F\left(t, \tau\left(\delta_{n}(t)\right) f_{n}^{x_{n}^{s}(t)}\right) \text { a.e. on }[0, T] \\
x_{n}^{s}(t)=\varphi_{0}(0)+\int_{0}^{t} \dot{x}_{n}^{s}(w) d w \forall t \in[0, T] \\
x_{n}^{s}(t) \in \Gamma(t) \forall t \in[0, T] \\
x_{n}^{s}(t)=\varphi_{0}(t) \forall t \in[0, T] .
\end{array}\right.
$$

By passing to the limit in (36) we obtain the existence of subsequence $\left(x_{n}^{s}\right)_{n}$ still denoted $\left(x_{n}^{s}\right)_{n}$ which converges to an absolutely continous function $x^{s}$ and $\dot{x}_{n}^{s}$ converges $\sigma\left(L^{1}, L^{\infty}\right)$ to $\dot{x}^{s}$. Similarly, we have

$$
\lim _{n} \tau\left(\delta_{n}(t) f_{n}^{x_{n}^{s}(t)}\right)=\tau(t-s) x^{s} \quad \forall t \in[0, T] .
$$

Because $F$ has a closed graph then we obtain $x^{s}(t) \in \Gamma(t) \quad \forall t \in[0, T]$ and $x^{s}$ is a solution of

$$
\left\{\begin{array}{l}
\dot{x}(t) \in F(t, \tau(t-s) x) \text { a.e. on }[0, T] \\
x(t)=\varphi_{0}(0)+\int_{0}^{t} \dot{x}(w) d w \forall t \in[0, T] \\
x(t) \in \Gamma(t) \forall t \in[0, T] \\
x(t)=\varphi_{0}(t) \forall t \in[0, T] .
\end{array}\right.
$$




\section{Conclusion}

In the present work, using a set-valued version of Scorza-Dragoni theorem, we weaken the regularity assumptions for the existence of viable solution for first order differential inclusions without delay. Then, we use this result to prove existence results of viable solutions for functional differential inclusions by means of a discretization approach. The viability set is more natural and general than those used previously. We treat the case of a fixed invariance set as well as the variable case.

\section{REFERENCES}

1. J.P. Aubin, Viability theory, Springer-Verlag, Boston, 1991.

2. H. Benabdellah and A. Faik, Perturbations convexes et nonconvexes des équations d'évolution, Portugaliae Mathematica, vol. 53, no. 2, pp. 187-208, 1996.

3. H. Benabdelah, C. Castaing and M. A. G. Ibrahim, BV solutions of multivalued differential equations on closed moving sets in Banach spaces, Geometry in Nonlinear Control and Differential Inclusions, vol. 32, pp. 53-81, 1995.

4. C. Castaing, M. A. G. Ibrahim and M. F. Yarou, Some contributions to nonconvex sweeping process, Journal of Nonlinear and Convex Analysis, vol. 10, no. 1, pp. 1-20, 2009.

5. C. Castaing, M. A. G. Ibrahim and M. F. Yarou, Existence problems in second order evolution inclusions: discretization and variational approach, Taiwanese Journal of Mathematics, Vol. 12, no. 6, pp. 1433-1475, 2008.

6. C. Castaing and M.D.P. Monteiro Marques, A multivalued version of Scorza-Dragoni's theorem with an application to normal integrands, Bulletin of the Polish Academy of Sciences, Mathematics, vol.42, pp. 133-140, 1994.

7. C. Castaing, M. Moussaoui and A. Syam, Multivalued differential equations on closed convex sets in Banach spaces, Set-Valued Analysis, vol.1, pp. 329-353, 1994.

8. C. Castaing, M. Valadier, Convex analysis and measurable multifunctions, Lecture Notes in Math. 580, Springer-Verlag, Berlin 1977.

9. K. Deimling, Multivalued Differential Equations, Walter de Gruyter, Berlin, New York, 1992.

10. G. Haddad, Monotone viable trajectories for functional differential inclusions, Journal of Differential Equations, vol. 42, pp. 1-24, 1981.

11. W. Jachimiak Viability for functional differential equations, Bulletin of the Polish Academy of Sciences Mathematics, vol. 42, no.1, pp. 55-61, 1994.

12. E. Mitidieri and I.I. Vrabie, Existence for nonlinear functional differential equations, Hiroshima Mathematical Journal, vol. 17, pp. 627-649, 1987.

13. A. Syam, Contribution aux inclusions différentielles, Thesis. Université de Montpellier II, 1993.

14. M. F. Yarou Discretisation methods for nonconvex differential inclusions, Electronic Journal of Qualitative Theory of Differential Equations, vol.12, pp. 1-10, 2009. 